\title{
El ciber-rumor y cybergossip en Colombia: una reflexión desde la ciberpsicología y las ciencias sociales computacionales ${ }^{\star 16}$
}

\author{
Harvey Mauricio Herrera-López \\ PhD. en Ciencias Sociales y Jurídicas \\ Universidad de Nariño, Colombia \\ Correo electrónico: mherrera@udenar.edu.co
}

Eva María Romera Félix

PhD. en Psicología

Universidad de Córdoba, España

Correo electrónico: eva.romera@uco.es

Rosario Ortega-Ruiz

PhD. en Psicología

Universidad de Córdoba, España

Recibido: 09/03/2020

Evaluado: $30 / 03 / 2020$

Correo electrónico: ed1orrur@uco.es

Aceptado: 28/04/2020

\section{Resumen}

Recientes estudios sugieren que el aumento del uso de las TIC y en especial de redes sociales parece estar relacionado con un mayor riesgo de exposición a fenómenos de intimidación en el ciberespacio. El ciber-rumor y el cybergossip son algunos de estos riesgos de gran relevancia en el estudio de la ciberconducta, pues modulan los procesos emocionales y actitudinales que pueden afectar negativamente al bienestar psicológico, así como al contexto social inmediato. Este documento presenta una revisión y reflexión general sobre el avance en el cuerpo teórico del cybergossip, delimita el constructo en comparación con el ciber-rumor y el cyberbullying, presenta un estudio realizado en Colombia desde una perspectiva cross-cultural y ofrece posibles líneas de trabajo futuras desde las ciencias sociales computacionales. Las conclusiones son útiles para los diversos estudios que abordan el estudio de la ciberconducta, particularmente desde la multidisciplinariedad y las ciencias sociales computacionales.

Cybergossip, ciber-rumor, cyberbullying, redes sociales, Colombia.

16 Para citar este artículo: Herrera-López, M., Romera, E. M., \& Ortega-Ruiz, R. (2021). El ciber-rumor y cybergossip en Colombia: una reflexión desde la ciberpsicología y las ciencias sociales computacionales. Informes Psicológicos, 21(2), pp. 259-280 http://dx.doi.org/10.18566/infpsic.v21n2a16

* Artículo resultado de los proyectos "El ciberodio, una nueva forma de violencia relacional. Un análisis explicativo desde la empatía y el desligamiento moral de jóvenes universitarios en San Juan de Pasto, Colombia", financiado por la Vicerrectoría de Investigaciones e Interacción Social-VIIS (Acuerdo No 213 de 2020), Universidad de Nariño, Pasto, Nariño (Colombia); y el proyecto "Competencia sociomoral y ecología del grupo de iguales en la violencia entre escolares: un estudio longitudinal y transaccional" (Ref. PSI2016-74871-R), financiado por el Ministerio de Ciencia e Innovación de España, Plan I+D+l. 


\title{
Cyber-rumor and cybergossip in Colombia: a reflection from cyberpsychology and computational social sciences
}

\begin{abstract}
Recent studies suggest that the increase in the use of ICT and especially of social networks seems to be related to a greater risk of exposure to bullying phenomena in cyberspace. Cyber-rumor and cybergossip are some of these highly relevant risks in the study of cyberbehavior, as they modulate emotional and attitudinal processes that can negatively affect psychological well-being, as well as the immediate social context. This document presents a general review and a reflection on the progress in the theoretical body of cybergossip. It defines the construct in comparison with cyber-rumor and cyberbullying, presents a study carried out in Colombia from a cross-cultural perspective and offers possible future lines of work from computational social sciences. The conclusions are useful for the various investigations that address the study of cyberbehavior, particularly from multidisciplinarity and computational social sciences.
\end{abstract}

Keywords

Cybergossip, cyber-rumor, cyberbullying, social networks, Colombia.

\section{Ciber-rumor e cibergossip na Colômbia: uma reflexão a partir da ciberpsicologia e das ciências sociais computacionais}

Resumo

Estudos recentes sugerem que 0 aumento do uso das TIC e principalmente das redes sociais parece estar relacionado a um maior risco de exposição aos fenômenos de bullying no ciberespaço. 0 ciber-rumor e 0 cybergossip são alguns desses riscos altamente relevantes no estudo do cibercomportamento, pois modulam processos emocionais e atitudinais que podem afetar negativamente 0 bem-estar psicológico, bem como 0 contexto social imediato. Este documento apresenta uma revisão geral e uma reflexão sobre os avanços no corpo teórico da cybergossip, delimita o construto em comparação com 0 cyber-rumor e 0 cyberbullying, apresenta um estudo realizado na Colômbia a partir de uma perspectiva transcultural e oferece possiveis linhas de trabalho futuro das ciências sociais computacionais. As conclusões são úteis para os vários estudos que abordam o estudo do cibercomportamento, nomeadamente da multidisciplinaridade $\mathrm{e}$ das ciências sociais computacionais. 


\section{ntroducción}

Las redes sociales y las aplicaciones (app) de mensajería instantánea se han convertido en mecanismos virtuales que han dotado de nuevas particularidades al surgimiento, desarrollo y mantenimiento de diferentes tipos de relaciones interpersonales, que engloban múltiples comportamientos desplegados a través de las tecnologías de la información y la comunicación (Davenport, Bergman, Bergman, \& Fearrington, 2014; Ellison, Vitak, Gray, \& Lampe, 2014). Es conocido por todos que el uso de las redes sociales está muy extendido y forma parte integral de la vida social de adolescentes, adultos y, cada vez más, de niños y niñas. Al respecto, recientes estudios resaltan que, con la creciente popularidad de las redes sociales, se ha creado un entorno que puede promover la difusión de comentarios dirigidos hacia personas o grupos en particular o para difundir rumores o información falsa de una manera más rápida y amplia que nunca, lo que puede tener repercusiones generalizadas para diversos grupos sociales (Eddine, Li, \& Ahmad, 2020).

Las diferentes aplicaciones de las redes sociales y mensajería instantánea permiten a los usuarios interactuar socialmente entre ellos, así como publicar y compartir información personal y social entre seguidores y amigos, como es el caso de Facebook, Instagram, Tuenti, Twitter, WhatsApp, Telegram, WeChat, entre otros (Chang \& Chen, 2014; Reinecke \& Trepte, 2014). Estas aplicaciones en línea se caracterizan por tener un perfil público, semipúblico o privado (Oh, Ozkaya, \& LaRose, 2014; Reinecke \& Trepte, 2014). Se suma a esto el hecho de que en la actualidad la mayoría de las aplicaciones de mensajería instantánea como WhatsApp, Telegram, Instagram, Messenger permiten que los mensajes, fotos, videos y grabaciones se compartan y difundan de manera inminente y en tiempo real, es decir "en vivo".

Información relacionadaconel proyecto Net Children Go Online (EU Kids Online), que estudia el impacto, la importancia de la seguridad en línea y otras condiciones referidas al uso de Internet entre niños y niñas, resalta que el uso de redes sociales es la actividad que más tiempo copa la atención de niños entre 9 y 12 años; así pues el 27\% de los niños de 9 a 10 años y el 60\% de los niños de 11 a 12 años tienen un perfil de red social, a pesar de que la presencia de menores en muchas redes sociales está legalmente restringida o prohibida hasta los 16 años o más (Mascheroni \& Cuman, 2014). Además, como lo indican Livingstone, Haddon, Vicent, Mascheroni y Ólafsson (2014), el 41\% de los que tienen entre 9 y 16 años utilizan asiduamente servicios de mensajería instantánea. En el caso de Colombia, a pesar de carecerse de cifras actualizadas, información referida por el Ministerio de Tecnología y Comunicación y algunas revistas y periódicos interesados en el tema, afirman que en los últimos años se ha registrado un incremento exponencial en la cantidad de usuarios de las redes sociales. Redes como Facebook y Twitter son las que gozan de mayor popularidad. Facebook se lleva el primer lugar con cifras cercanas a los 20 millones de usuarios, siendo Bogotá 
la novena ciudad del mundo con mayor número de perfiles (cerca de 8 millones). Twitter tiene alrededor de 7 millones de perfiles colombianos y cada vez más se convierte en el medio preferido para desplegar información relacionada con multiplicidad de temas; estas cifras ubican a Colombia por encima de países como Francia y Alemania. Una reciente publicación del periódico La República (Cigûenza, 2019), a partir de datos ofrecidos por ComScore, Cintel y la Interactive Advertising Bureau Colombia (IAB), compañías de investigación de marketing en internet que incluye el estudio del comportamiento de usuarios de internet a nivel mundial, refiere que el alcance de las redes y medios sociales de comunicación por internet es del 83.8\%; esta lista de países de América Latina la lidera Perú con un 93.2\%, Brasil con $89.1 \%$, México con 87.8\%, Argentina con 83.8\%, luego Colombia y, finalmente, Chile con $82.9 \%$. Se resalta igualmente que los equipos o dispositivos de mayor uso y acceso a la cotidianidad, principalmente de la casa u hogar, son el smartphone con un $72 \%$, seguido por el portátil y/o computador de escritorio con un 38\% y por la TV inteligente (Smart tv) con un 32\%. Se reconoce además que las actividades más frecuentemente realizadas por los usuarios de estos dispositivos son el uso de redes sociales en un $88 \%$, el chat en $86 \%$, el envío de correos electrónicos con el $79 \%$ y el compartir videos o imágenes con $78 \%$. Es de notable importancia mencionar que, en promedio, el colombiano utiliza 6.7 horas al mes para navegar en redes sociales, siendo los hombres, con un 53\%, los que más las usan. Respecto a la edad, cabe destacar que son los adolescentes jóvenes entre 15 y 20 años los que más usan las redes sociales (29\%), seguidos por las personas con edades entre 25 y 34 años (24\%).

Lo anterior permite reconocer que las nuevas tecnologías y las aplicaciones diseñadas para la interacción en las redes sociales, crean un sistema multidireccional que puede favorecer los procesos de comunicación maximizando la influencia de algunos mensajes, los cuales, por lo general, buscan la propagación de información positiva; no obstante, las cosas indeseables, como rumores, "memes" ofensivos, noticias falsas, entre otras, también pueden propagarse de manera similar y podrían producir daños significativos (Yang, Li, \& Giua, 2020). Un ejemplo de esto fue el rumor "dos explosiones en la Casa Blanca y Obama está herido", que ocurrió el 23 de abril de 2013; noticia falsa que causó pérdidas de 10 mil millones de dólares antes de que se aclarara el rumor (Peter, 2013).

Si bien es posible asumir que en los últimos años se ha visto un gran avance en los mecanismos de comunicación, especialmente de la mano del desarrollo vertiginoso de los dispositivos electrónicos, este desarrollo y el uso más frecuente de las redes sociales y demás medios digitales de comunicación, ha incrementado el nivel de exposición de los usuarios, tanto a eventuales situaciones de agresión, como a la masiva recepción de información que no corresponde a la verdad y que genera cadenas de desinformación (rumores o chismes); se suma a lo anterior la exposición a innumerables mensajes comerciales o de marketing que llenan todo tipo de correos electrónicos personales e institucionales. 


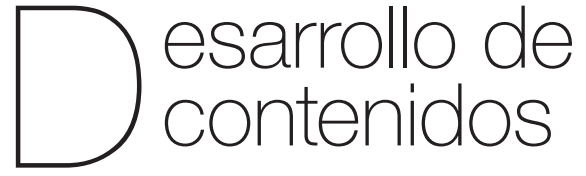

\section{Rumor y Gossip}

Los rumores son mensajes circulantes en las conversaciones de la gente acerca de lo que concierne al interés general y público, de acuerdo con la actualidad, cuya característica es ser una voz paralela, cuando no opuesta, a las fuentes institucionalmente autorizadas. El rumor aparece en la colectividad como una información que no ha sido confirmada por las fuentes oficiales o que éstas han desmentido. Así, el rumor adquiere funciones que impactan los procesos de desinformación, inestabilidad, ruptura o creación de nuevos contextos (Kapferer, 1989).

Schindler (2003) refiere que los rumores se pueden clasificar en dos grandes grupos: 1) los que surgen de manera espontánea y 2) los que surgen de manera deliberada o intencional; así mismo, complementariamente reconoce tres fuentes de origen como son: a) los que surgen de eventos genéricos, b) de eventos específicos y/o detalles y c) los que son producto de la fantasía. Si bien el estudio del rumor ha sido objetivo frecuente de la psicología y la sociología, existen estudios desde ámbitos particulares como la economía y las finanzas que evidencian el gran impacto de este fenómeno. Es frecuente, por tanto, encontrar grandes inversiones por parte de muchas empresas en el mundo globalizado para implementar programas en los medios sociales y de comunicación para protegerse de amenazas latentes contra la confianza de sus clientes, la información referida a la marca y la construcción de su imagen y reputación (Tinoco, 2004).

El gossip, por su parte, se define como un tipo de conversación de carácter evaluativo, generalmente entre personas que tienen familiaridad entre sí, sobre terceras personas que no están presentes (Eder \& Enke, 1991; Foster, 2004). Estas conversaciones son situacionales, por lo que la emisión del mensaje y su interpretación dependen del contexto en el que se produzcan (Wert \& Salovey, 2004). Se reconoce que el gossip es una práctica antigua y muy extendida, a la que dedicamos alrededor de dos tercios de nuestras conversaciones diarias (Dunbar, Duncan, \& Marriott, 1997). A pesar de ello, este comportamiento cuenta con una mala reputación en todas las épocas y culturas porque es considerado una práctica egoísta realizada para manipular maliciosamente las impresiones que tienen los demás sobre una tercera persona (Goodman, 2000). No obstante, existen algunos estudios de revisiones teóricas sobre esta práctica que contradicen esta tendencia y sostienen una perspectiva más positiva del gossip (Baumeister, Zhang, \& Vohs, 2004; Dunbar, 2004). Esto es posible y se explica porque en su significado y acepción inglesa no tiene necesariamente una connotación negativa, pues alude al compartir información e impresiones con las personas más cercanas; mientras que la traducción al castellano surge desde las palabras latinas schisma/cimex que significan división o separación denotando un originario carácter negativo (Pietrosemoli, 2009). Desde este argumento es importante aclarar que, dado que la palabra traducida al castellano no corresponde al alcance positivo mencionado en los estudios, en este manuscrito se ha decidido utilizar la acepción inglesa gossip. 
En el marco de una perspectiva amplia se evidencian investigaciones que se han centrado principalmente en las funciones y beneficios grupales del gossip, como son: a) informar a sus miembros sobre las normas y sanciones (Grosser, Lopez-Kidwell, \& Labianca, 2010), b) proteger al grupo de aquellos que violan las normas (Beersma \& Van Kleef, 2011; Feinberg, Willer, \& Schultz, 2014), c) influir en la reputación de sus integrantes (Giardini, 2012) y d) fortalecer los vínculos sociales (Dunbar, 2004). De esta manera, se puede afirmar que el gossip, en términos generales, puede promover el bien común (Beersma \& Van Kleef, 2011). Al respecto, algunos estudios subrayan precisamente que el gossip también puede servir para restringir los comportamientos egoístas que perjudican a un grupo gracias a la fuerte influencia que tiene sobre la reputación y la inclusión de sus miembros; esta práctica recientemente se ha denominado "chisme inverso" que significa en esencia hablar bien de los demás (Beersma, \& Van Kleef, 2011).

A pesar de que se ha avanzado en la comprensión del gossip y sus posibles beneficios grupales, relevantes investigaciones subrayan que también tiene beneficios individuales para quien lo recibe, porque permite obtener información evaluativa por parte de los otros para evaluarse a uno mismo; necesidad básica que se ve satisfecha a través de la comparación social indirecta (Wert \& Salovey, 2004). Concretamente, se ha evidenciado que el gossip positivo promueve un valor agregado de superación personal, que es una importante dimensión de la autoevaluación (Martinescu, Janssen, \& Nijstad, 2014). Este propósito hacia la mejora del individuo se relaciona con la función de aprendizaje que provee el gossip, pues aporta algunas claves importantes sobre cómo tener éxito o evitar fracasos en situaciones sociales concretas (Baumister et al., 2004). Desde esta perspectiva, los individuos están más interesados en recibir gossip positivo pues representa modelos a replicar de comportamiento social (Litman \& Pezzo, 2005).

Es de resaltar que, a pesar de que aún existen debates sobre su definición, se reconoce que el gossip, en un sentido general, incluye comentarios sociales que no necesariamente son negativos sobre terceras personas y que eventualmente puede proporcionar información útil y estrechar vínculos sociales en algunos grupos. Además, tiene una función individual gratificante y es una de las mejores formas de lograr entretenimiento grupal (Foster, 2004). De hecho, se ha evidenciado que lo que diferencia al gossip de la "curiosidad social" es su objetivo; mientras que el primero responde predominantemente a fines de entretenimiento, la curiosidad social se dirige en mayor medida al interés en obtener información sobre otras personas (Hartung \& Renner, 2013).

El gossip implica una serie de intenciones que se develan al interpretar el mensaje; por lo tanto, tiene diferentes funciones o intenciones específicas: a) informar, es decir, difundir información a otras personas; b) entretener y disfrutar de esta actividad; c) construir amistades y mantenerlas a través de una comprensión compartida de los estándares sociales; además, aporta al fomento de vínculos que impliquen cercanía y confianza; y d) influir, es decir, el gossip se usa como un medio para interferir en la forma en que los oyentes reaccionan y ven a otras personas (Foster, 2004). El cumplimiento de estas funciones exige una serie de pasos que 
obedecen a la estructura básica del fenómeno: en un primer momento se identifica o focaliza a la persona, objetivo del chisme (generalmente ausente del contexto de comunicación), luego se ejecuta la elaboración y despliegue del contenido del gossip dotándolo de una intencionalidad (negativa o positiva), a continuación se procede a la recepción por parte de los oyentes, simultáneamente se procede a la decodificación, interpretación y evaluación de lo que se comunica (no obstante, es posible también que se perciba como carente de intencionalidad) y, finalmente, se despliegan las respuestas que puede incluir: la aceptación del mensaje (dando credibilidad al emisor), la expansión de la comunicación, la solicitud de aclaración o la reacción en oposición del espectador u oyente (Eder \& Enke, 1991).

\section{Ciber-rumor y cybergossip}

Un estudio reciente con más de 55000 adolescentes de 20 países diferentes subraya el extendido uso que se hace de las TIC para la comunicación social y la importancia que tienen para su desarrollo personal y social (Areepattamannil \& Khine, 2017). A pesar de disponer de amplia evidencia empírica y teórica referida a la función socializadora del uso de las TIC en las relaciones de los adolescentes, resulta llamativo que se le haya prestado más atención investigadora al gossip y sean escasos los trabajos dirigidos al cybergossip, que es la misma conducta pero desplegada a través de las TIC (Laghi et al., 2013; Oluwole, 2009; Subramanian, 2013). Es notorio además, que la mayoría de estas investigaciones se hayan realizado en el campo de las Ciencias de la Computación (Apolloni, Malchiodi, \& Taylor, 2013), siendo muy pocas las que se han realizado desde la psicología (Gabriels \& De Backer, 2016).

Si bien el cybergossip parte de la definición del gossip, ambos fenómenos deben ser estudiados de forma diferenciada porque sus formas de presentación se ajustan a las peculiaridades de los escenarios por los que se difunden los comentarios sobre terceras personas. Al respecto es necesario resaltar: 1) el gossip se despliega "cara a cara" y hace uso del lenguaje verbal, mientras que el cybergossip utiliza mensajes escritos, que se acompañan, en gran medida, por imágenes y vídeos que apoyan la supuesta o probada veracidad de lo que se está diciendo, 2) en el cybergossip las palabras quedan registradas o grabadas y pueden ser remitidas a otras personas, característica que influye en el proceso de planificación del contenido y en su impacto posterior, pues todo lo que se "sube" al internet es imborrable y queda a disposición de cualquier persona (Bertolotti \& Magnani, 2013), 3) no es comparable la posibilidad de compartir la información con una amplia red en cualquier momento, ni la velocidad de su transmisión en el contexto on-line que en el offline, y 4) la comunicación virtual dificulta la posibilidad de que el emisor trasmita su intencionalidad social y que el receptor disponga de mayores recursos para interpretarla, pues carece de elementos prosódicos, paralingüísticos y extralingüísticos que favorecen la comprensión de la información y de las emociones presentes en las interacciones (Carrera \& Pelayo, 2002). Esta carencia aumenta la posibilidad de que surjan malentendidos que pueden desembocar en conductas 
de ciberagresión y cibervictimización entre los adolescentes, propias del cyberbullying (Del Rey et al., 2015).

La posibilidad de que el cibergossip influya negativamente en un grupo ha llevado a que numerosos estudios lo identifiquen como una forma indirecta de agresión entre iguales, pues a partir de su práctica, se busca manipular la reputación de otro o su exclusión del grupo (Archer \& Coyne, 2005). Esta afirmación se sustenta a partir de lo que Mason (2008) conceptualiza como ciberagresión:

\begin{abstract}
Es la conducta de un individuo o un grupo que usa intencionalmente información y comunicación, involucrando tecnologías electrónicas, para facilitar el acoso o la amenaza deliberada y repetida sobre otro individuo o grupo, enviando o publicando textos crueles y/o gráficos (p.323).
\end{abstract}

En el cyberbullying se reconoce una clara intencionalidad de hacer daño a otra persona, lo cual generalmente se convierte en una práctica sistemática sobre una víctima que no puede defenderse por sí misma (Smith, 2006; Tokunaga, 2010). No obstante, los anteriores aspectos: intencionalidad, repetición/sistematicidad y desbalance de poder, no siempre están presentes en el cybergossip, lo cual sugiere que no son fenómenos equiparables, aunque están fuertemente relacionados. Es necesario enfatizar que la intencionalidad del gossip no está definida solo por el emisor, sino que el grupo o individuo receptor también puede definir el carácter intencional de los comentarios emitidos. Al respecto, Foster (2004) argumenta que la primera respuesta es más importante que el propio comentario de evaluación, pues va a determinar la dirección del resto de la conversación.

Es posible que los comentarios hechos sobre otras personas provoquen una actitud de rechazo por parte de quienes reciben la información, haciendo que reaccionen en oposición o agresivamente frente el autor o autores del cibergossip, y que se puedan convertir en potenciales víctimas. Este comportamiento también puede estar motivado por la impulsividad que ocurre en entornos interactivos pues internet permite a las personas responder de inmediato en cualquier lugar y en cualquier momento. Una explicación adicional de esta relación reside en el hecho de que los ciberagresores se sienten más motivados no solo a ser proactivos frente a estos problemas, sino también a tener un comportamiento reactivo en episodios de cibergossip (López-Pradas, Romera, Casas, \& Ortega-Ruiz, 2017).

Por lo argumentado, el cyberbullying y el cybergossip deben ser estudiados independientemente dadas sus características, así como la forma diferenciada y particular de sus comportamientos violentos, pues, a pesar de que hay evidencias relevantes sobre su interacción y relación, algunos estudios han reconocido que el cybergossip no siempre conduce a comportamientos típicos de ciberagresión y cibervictimización (López-Pradas et al., 2017).

\section{La medición del Cybergossip}

Abordar el estudio de la medición del cybergossip requiere apelar inicialmente a las investigaciones sobre su versión 
off-line, es decir el gossip. El gossip ha sido ampliamente estudiado desde diferentes metodologías de investigación pero mayoritariamente se han utilizado técnicas de observación para su medición (Foster, 2004); sin embargo, dado que el gossip es un fenómeno ligado a la confianza y la discreción (Gluckman, 1963), no se recomiendan los estudios experimentales y observacionales, pues estos diseños hacen difícil garantizar ambas condiciones.

La revisión de evidencias empíricas refleja que los estudios cuantitativos son limitados, a pesar de la necesidad detectada de avanzar hacia el diseño de instrumentos de medida (Noon \& Delbridge, 1993). El uso de autoinformes, a pesar de sus inconvenientes, relacionados principalmente a la deseabilidad social, se convierte en un sistema de medición idóneo del gossip, pues su carácter anónimo y retrospectivo permite mantener la confianza y la discreción requeridas (Brady, Brown, \& Liang, 2017).

Las escasas escalas disponibles presentan una serie de limitaciones relacionadas, tanto con la definición de partida, como con el foco de estudio del instrumento. En el primero de los casos, existen escalas que conciben el gossip como un comportamiento negativo, e incluso lo identifican como un modo de agresión indirecta, como es el caso de la escala Direct and Indirect Aggression Scale -DIAS (Björkqvist, Lagerspetz, \& Österman, 1992) o la Indirect/Social/Relational Aggression scale -ISRA (Coyne, Archer, \& Eslea, 2006). En el segundo caso, los instrumentos que se ajustan a la definición de gossip desde una perspectiva amplia, la cual incluye su valor positivo además del negativo, se centran en aspectos concretos, que van desde el contenido de lo que se habla (Nevo, Nevo, \& Derech-Zehavi, 1993), a las actitudes (Litman \& Pezzo, 2005), motivaciones (Beersma \& Van Kleef, 2011) o funciones del gossip (Foster, 2004), pero no se dirigen a la medición del comportamiento en general (Brady et al., 2017).

Un instrumento de reconocido uso es el Tendency Gossip Questionnaire (Nevo et al., 1993), el cual fue validado con 120 estudiantes universitarios israelíes. Se trata de una escala tipo Likert, compuesta por 19 ítems (con siete opciones de respuesta) que aluden al contenido de las conversaciones (apariencia física, logros, información social y chismes sublimados). Esta escala es criticada precisamente por su dificultad para medir con unos pocos ítems la amplia variabilidad de contenidos que pueden abordarse en el gossip (Litman \& Pezzo, 2005).

Otro instrumento es el Gossip Functions Questionnaire (Foster, 2004), que mide el fenómeno con 24 ítems (tipo Likert) que engloban las cuatro funciones básicas identificadas en el gossip: información, amistad, influencia y entretenimiento. Estas funciones han servido de base para investigaciones posteriores, realizadas en su mayoría con estudiantes no graduados (Hartung \& Renner, 2013; Watson, 2012). La dificultad reconocida de esta escala es que no incluye al contexto donde se despliega este fenómeno.

También se encuentra el Motives to Gossip Questionnaire (Beersma \& Van Kleef, 2011) que supera en cierta medida las limitaciones mencionadas previamente. Este instrumento está conformado por 22 ítems, agrupados en cuatro dimensiones (informar, entretenerse, influir 
y mantener las normas del grupo), las cuales valoran los motivos por los que los jóvenes universitarios se implicaron en la última situación de gossip. El problema de este instrumento es que solo valora la presencia de la conducta de gossip y no su frecuencia. Por otro lado, contempla que la motivación de influencia en el gossip tiene una dirección negativa, siendo relacionada con la agresión indirecta. Al respecto, los estudios originales de Stirling (1956) y Foster (2004) reconocen que esta influencia puede tener además un sentido positivo, el cual está en la base del aprendizaje de la cultura de los miembros de un grupo.

Coincidiendo con Brady et al. (2017), se hace necesario un adecuado registro de la frecuencia de comportamiento para solventar la falta de generalidad en la naturaleza del constructo e incluso los posibles sesgos observados en su medición. Estos autores han validado recientemente la escala Workplace Gossip, que recoge la frecuencia con la que se ha realizado el gossip en el contexto laboral, con ítems y dimensiones específicas para este campo de estudio.

Resulta destacable que las escalas en mención han sido diseñadas y validadas para jóvenes y adultos. En las investigaciones con niños y adolescentes, el gossip ha sido estudiado principalmente a través de estudios narrativos y observacionales, y se engloba dentro del repertorio de conductas agresivas indirectas que se realizan para hacer daño a otros (Xie, Cairns, \& Cairns, 2005). Los resultados de los estudios con perspectiva del desarrollo humano reconocen que, a medida que crecen, los chicos y chicas ejercen en mayor medida agresiones indirectas, como el gossip, que utilizan para victimizar a sus iguales (Archer \& Coyne, 2005). En los estudios etnográficos, las chicas adolescentes son representadas en mayor medida como ejecutoras del gossip, utilizándolo para mantener su estatus social (Merten, 1997), no obstante, recientes revisiones sistemáticas subrayan que la relación entre el género femenino y el gossip, entendido en su versión maligna, es un estereotipo de género (McAndrew, 2014). En esta línea, algunos meta-análisis subrayan que las diferencias de género en las formas de agresión indirecta, incluyendo el gossip, son inconsistentes (Archer, 2004; Card, Stucky, Sawalani, \& Little, 2008).

Al igual que con jóvenes y adultos, los estudios con adolescentes necesitan avanzar hacia la consideración del gossip como una conducta que promueve el aprendizaje social, que favorece la comprensión de las normas del grupo y que forma parte del repertorio de conductas socialmente competentes (McDonald, Putallaz, Grimes, Kupersmidt, \& Coie, 2007); esta apreciación se corrobora con investigaciones que refieren resultados positivos del gossip en las edades infantiles (Engelmann, Herrmann, \& Tomasello, 2016). Los avances en esta línea apuntan hacia la necesidad de hacer explícitas las valoraciones morales de los que practican el gossip (gossipers), para regular las relaciones y generar mayor confianza ( $\mathrm{Pe}$ ters \& Kashima, 2015). Este tipo de estudios se hacen particularmente relevantes en la adolescencia porque en esta etapa se consolidan los estilos de interacción en relaciones cercanas. Identificar y describir los comportamientos que caracterizan estos estilos de relación se convierte en un objetivo de gran interés investigador, que permitirá predecir importantes aspectos del funcionamiento social y personal en la 
adultez (Perry \& Pauletti, 2011). Se requiere por tanto, el uso de instrumentos elaborados para adolescentes que incorporen los avances en la medición del gossip en edades juveniles y adultas, y que se adecuen a las distintas formas de interacción, donde las tecnologías de la información y la comunicación (TIC) sean consideradas con relevancia, pues han conllevado notables cambios en las relaciones sociales de los chicos y chicas (Lee, Kim, Golden, Kim, \& Park, 2016).

Para dar respuesta a la necesidad detectada de contar con escalas que contemplen los comentarios sobre terceras personas que chicos y chicas realizan a través de las redes, López-Pradas et al. (2017) diseñaron y validaron una escala de cybergossip denominada Cybergossip-Q-Primary - CGQ-P para escolares de 10 a 12 años, ajustada a los criterios identificados en la literatura científica en cuanto a su definición y medición (LópezPradas et al., 2017). Este instrumento incluye nueve tipos de comportamientos que engloban las funciones identificadas en el gossip (influencia, entretenimiento, información y amistad). La escala aborda el estudio del fenómeno desde una perspectiva amplia que incluye la realización de comentarios negativos y positivos que pueden promover o dificultar las relaciones entre iguales e incluye además la medida de frecuencia de dichas conductas. Las óptimas propiedades psicométricas del instrumento permiten contar con una escala de cybergossip para niños y niñas de escuela primaria.

Recientemente se publicó la validación de la escala CGQ-P de López-Pradas et al., (2017) en adolescentes escolarizados, incluyendo una muestra de españoles y colombianos; así, esta escala pasó a denominarse Cybergossip-Q-Adolescents -CGQ-A (Romera, Herrera-López, Casas, Ortega, \& Del Rey, 2018). Este estudio confirmó la estructura unidimensional de la escala original con óptimos índices de ajuste y consistencia interna. Así mismo, los resultados de validez discriminante mostraron que el CGQ-A es una medida diferente a la de ciberagresión y cibervictimización, aunque guarda cierta relación. Estos resultados contradicen la conceptualización reducida del fenómeno como un comportamiento malicioso y son consistentes con la investigación que aborda el estudio del cibergossip desde una perspectiva amplia (Beersma \& Van Kleef, 2011; Wert \& Salovey, 2004).

Los análisis multigrupo entre la muestra de Colombia y España, y entre chicos y chicas, reflejaron que la escala responde a una estructura factorial robusta y homologable a ambos países y al género. Las medidas de invarianza de la escala obtenidas entre adolescentes colombianos y españoles indican una conceptualización similar del cybergossip cuando responden a la escala CGQ-A, lo que señala que existen dinámicas similares en los dos países, a pesar de las diferencias culturales, socioeconómicas y geográficas. El mismo análisis de invarianza de la estructura de la prueba fue aplicada al género, sin encontrarse diferencias, lo que sugiere que se está midiendo un fenómeno identificado del mismo modo por chicos y chicas (Romera et al., 2018). Aunque el cibergossip ha sido ampliamente identificado como un comportamiento universal (Dunbar, 2004), este es el primer estudio que mide el carácter cros-cultural del fenómeno en adolescentes, en este caso, realizado a través de las TIC. En general, estos hallazgos contradicen los trabajos que identifican el cibergossip 
con la agresión indirecta, en los que se reconoce que ellas identifican en mayor medida este tipo de estrategias sociales manipulativas como una forma de agresión (Archer \& Coyne, 2005). En cambio, soportan las investigaciones que afirman que el cibergossip, entendido en sentido amplio, es una conducta reconocida e identificada tanto por ellos como por ellas (Kuttler, Parker, \& La Greca, 2002).

\section{Cybergossip en Colombia}

Es oportuno enfatizar que, para el caso de Colombia, el estudio de Romera et al. (2018) es el primer estudio que permite conocer la frecuencia del fenómeno atendiendo a las diferencias de país y género. Este estudio incluyó 1931 adolescentes de los cuales 46.9\% fueron hombres y $53.1 \%$ mujeres, todos escolarizados en colegios públicos y privados del sur del país, con edades entre 10 y 19 años $(M=14.22 ; S D=1.89)$. Dentro de los resultados es oportuno destacar que el $85.6 \%$ ( $n=1653$ ) reportaron tener red wifi en casa. Así mismo, el 80.7\% ( $\mathrm{n}=$ 1558) de los estudiantes poseen teléfono móvil (celular); de estos el 30.3\% ( $n=472$ ) disponen de datos móviles para navegar por internet. Respecto al tiempo de uso de las redes sociales, el 7.7\% $(n=148)$ de los estudiantes manifestaron no usar las redes sociales, el 48.34\% ( $n=934)$ las usan menos de 3 horas al día, el 28.4\% ( $n$ $=548$ ) navegan en ellas entre 3 y 6 horas al día y el $15.6 \%$ ( $n=301)$ pasan más de 6 horas al día en redes sociales. Lo anterior permite reconocer que en un gran porcentaje los adolescentes participantes del estudio interactúan y se relacionan a través del internet, más específicamente a través de las redes sociales. Además, se reconoce que hay un porcentaje no despreciable de adolescentes escolarizados que dedican alrededor de la mitad de la jornada del día para navegar por las redes sociales; esto pone de manifiesto la necesidad de fortalecer: a) la información y cualificación sobre los riesgos a los cuales están expuestos, p.e. cibervictimización, grooming, sexting, entre otros; b) la posibilidad de aprovechar el tiempo de uso del internet, quizás para efectos más educativos; y c) las potenciales dinámicas de adicción que pueden alterar o interferir en las actividades o responsabilidades off-line como lo son las familiares, académicas e inclusive sociales.

En referencia al cybergossip, la conducta que más frecuentemente se presenta es la de hablar sobre las demás personas por medio de las redes sociales, con el objetivo de sentirse más cerca o cohesionado a un grupo; seguido por hablar sobre un compañero o amigo por las redes sociales para que el grupo cambie su opinión sobre él (ver Tabla 1).

Tabla 1.

Frecuencias de respuesta de adolescentes colombianos a los ítems del cuestionario CGQ-A

\begin{tabular}{lccc}
\hline \multicolumn{1}{c}{ Ítem } & frec \% hombre (chico) & frec \% mujer (chica) & frec \% total \\
\hline $\begin{array}{l}\text { He hecho comentarios de otros compañeros para con- } \\
\text { seguir estar dentro de un grupo en las redes sociales. }\end{array}$ & 1.5 & .2 & 1.1 \\
$\begin{array}{l}\text { Hablo sobre los demás por las redes sociales, porque } \\
\text { me hace sentir más cerca de mi grupo. }\end{array}$ & 6.1 & 4.1 & 5.1 \\
\hline
\end{tabular}




\begin{tabular}{|c|c|c|c|}
\hline Ítem & frec $\%$ hombre (chico) & frec \% mujer (chica) & frec $\%$ total \\
\hline $\begin{array}{l}\text { He hablado sobre un compañero o amigo por las redes } \\
\text { sociales, para que el grupo cambie su opinión sobre él. }\end{array}$ & 6 & 2.9 & 4.3 \\
\hline $\begin{array}{l}\text { Cuando me enfado o peleo con un compañero o amigo, } \\
\text { lo cuento en las redes sociales o Whatsapp. }\end{array}$ & 3.1 & 3.2 & 3.1 \\
\hline $\begin{array}{l}\text { He contado cosas malas sobre otras personas por las } \\
\text { redes sociales sin darme cuenta. }\end{array}$ & 4.2 & 3.4 & 3.7 \\
\hline $\begin{array}{l}\text { He contado secretos que me ha dicho un compañero(a) } \\
\text { por las redes sociales. }\end{array}$ & 3.4 & 2.4 & 2.8 \\
\hline $\begin{array}{l}\text { Cuento las cosas que me entero de otros amigos por } \\
\text { las redes sociales o Whatsapp. }\end{array}$ & 5.1 & 3.3 & 4.1 \\
\hline $\begin{array}{l}\text { Cuando alguien de mi grupo hace algo que está mal, lo } \\
\text { cuento al resto de compañeros por las redes sociales. }\end{array}$ & 3.8 & 1.5 & 2.6 \\
\hline $\begin{array}{l}\text { Hablo en mi grupo de amigos de las redes sociales } 0 \\
\text { Whatsapp sobre los que les pasa a otros compañeros } \\
\text { del colegio para divertirme. }\end{array}$ & 4.8 & 2.8 & 3.7 \\
\hline
\end{tabular}

Nota: frec. $=$ frecuencia de respuesta, $\%=$ porcentaje

En general, los resultados descriptivos señalan que el cybergossip es una ciberconducta que los adolescentes ponen en práctica con cierta asiduidad y no necesariamente con una intención negativa. De igual manera, se reconoce que los adolescentes colombianos están menos implicados en cybergossip que los españoles. Estos resultados pueden interpretarse a razón del menor uso de las TIC por parte de la población colombiana (Said-Hung, 2014), pero también podría deberse a las propias diferencias entre las culturas. La cultura española se caracteriza por una mayor promoción de valores individualistas relacionados con la imagen social, el reconocimiento y la aceptación dentro del grupo (Tafarodi \& Swann, 1996); de ahí quizás su mayor implicación en este fenómeno interactivo. En cambio, la cultura latinoamericana es más restrictiva, y se valora en mayor medida el respeto a la normas, la conformidad y la obediencia (Lila, Musitu, \& Buelga, 2000), lo que puede explicar una menor participación en comportamientos socialmente sesgados, como es el caso del cybergossip negativo.

Los resultados relacionados con el género señalaron que no existen diferencias entre chicos y chicas en la implicación en cybergossip. Al respecto, diferencias de género han sido reconocidas en estudios evolutivos los cuales subrayan que las adolescentes emplean más tiempo en las actividades sociales, mostrando un estilo de relación caracterizado por la cercanía y la ansiedad ante el rechazo (Perry \& Pauletti, 2011). Algunas investigaciones sobre agresividad indirecta, reconocen que las chicas están más implicadas (Archer \& Coyne, 2005), al igual que en el gossip (Nevo et al., 1993); sin embargo, los resultados de esta investigación muestran ausencia de estas diferencias, coincidiendo con la mayoría de las aportaciones en el campo de estudio del gossip y cibergossip en sentido amplio (Card et al., 2008; Foster, 2004). Este resultado tiene dos implicaciones: a) lleva a cuestionar la visión estereotipada del género femenino que 
conduce a dar por verdadera la creencia de que las chicas practican más el cibergossip que los chicos (tanto en el sentido amplio como reducido del concepto). Al respecto, algunos estudios atribuyen esta asociación a que los esquemas de género, construidos desde pequeños, tiendes a condicionar el desempeño en la interacción social; es decir, se asume que las niñas se ajustan más a un esquema de interacción verbal, mientras que los niños, interactúan predominantemente en un escenario físico o corporal (Card et al., 2008), y b) plantea la necesidad de incluir a los chicos en los estudios sobre gossip y cybergossip y no sesgar la muestra con la atribución de que el género femenino posee una mayor implicación (Massar, Buunk \& Rempt, 2012; McDonald et al., 2007).

Finalmente, es necesario enfatizar que el cybergossip no tiene por qué entenderse como una ciberconducta a erradicar o disminuir. Por el contrario, investigaciones recientes respaldan las posibles ventajas de incluir al cybergossip en intervenciones psicoeducativas destinadas a promover el aprendizaje de nuevos métodos de interacción y el desarrollo de competencias comunicativas y digitales para construir relaciones virtuales positivas y prevenir comportamientos cibernéticos riesgosos (Romera et al., 2018). No obstante, es importante no olvidar las posibles consecuencias negativas de este fenómeno para ayudar a mejorar las relaciones interpersonales, especialmente si se trata de niños con edades tempranas, como los son estudiantes de primaria, quienes están expuestos cada vez más al uso temprano de los dispositivos digitales. Esto tendría considerables repercusiones educativas, dado que la escuela es uno de los estamentos sociales llamado a enseñar y fomentar las competencias comunicativas y digitales entre sus estudiantes.

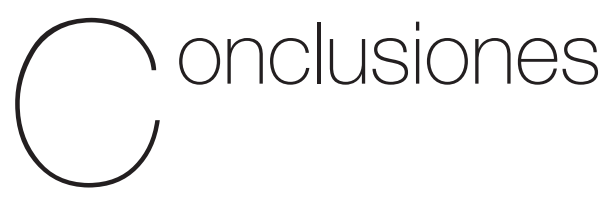

\section{El cybergossip desde la ciberpsicología y las ciencias sociales computacionales}

En la actualidad las personas se están uniendo crecientemente a múltiples redes sociales, en donde tienen cada vez más probabilidades de intercambiar, mensajes, ideas, compartir información, promover argumentos ideológicos, políticos y religiosos, entre otros, e incluso promover la compra de productos y servicios; en este escenario de vertiginosa interacción, los ciber-rumores y el cybergossip se propagan simultáneamente, creando así una nueva dimensión incluida en el complejo proceso de la interacción en red. El hecho de que actualmente existan más de 3000 millones de individuos conectados a internet y más de 7000 millones de suscriptores de telefonía celular en el mundo exige miradas integrativas desde las perspectivas de las ciencias sociales computacionales, la ciencia de datos y las humanidades digitales (Meneses, 2018). Esta nueva dimensión de interacción en red a través de dispositivos y aplicaciones digitales nos ha permitido cuantificar y registrar el comportamiento humano a gran escala, configurando lo que se denomina los small data (pequeños volúmenes) y el 
big data (grandes volúmenes) que es una radiografía de la inteligencia colectiva. En consecuencia, nos movemos en el mundo de los datos; datos que crecen de manera exponencial y que se convierten en fuente de información para los científicos sociales que buscan comprender de forma realista y crítica la transformación de datos en conocimiento útil para la sociedad. Esta tarea implica no solamente el diálogo con las ciencias computacionales, sino adquirir nuevos conocimientos, habilidades y lenguajes (Meneses, 2018).

A partir de lo mencionado anteriormente es posible sugerir que se requiere incluir estas nuevas miradas para continuar avanzando en la comprensión del gossip y el cybergossip, poniendo atención en las características del individuo, pero de manera más particular, en las del grupo, siguiendo las líneas de investigación recientes que subrayan la importancia de entender la influencia de las redes sociales en el comportamiento social (Wu, Balliet, \& Van Lange, 2016). Es necesario además continuar explorando en el rol del género en el cybergossip e indagando también en los posibles matices que variables relacionales como la amistad pueden introducir (Watson, 2012). Así mismo, resulta de interés abordar los factores personales (disponibilidad, habilidad, hábito, usos) y motivacionales (autoeficacia, interés, disfrute) que están relacionados con el uso de las TIC (Areepattamannil \& Khine, 2017). Se hace necesario igualmente desarrollar investigaciones de corte evolutivo que permitan analizar las diferencias de implicación en el gossip y el cybergossip.

Dada la estrecha relación entre la práctica del cybergossip y el ciber-rumor, se requiere analizar de manera precisa su interacción, pues con la creciente popularidad de las redes sociales en línea, se ha creado un entorno que permite difundir rumores de una manera más rápida y amplia que nunca, lo que puede tener repercusiones generalizadas en la sociedad y los individuos. Al respecto, se ha dedicado una gran cantidad de investigaciones al análisis de la propagación de información, opinión, comportamiento social e innovación. En este sentido, algunos autores proponen innovadoras estrategia de "campañas de verdad" para minimizar la influencia de los rumores en las redes sociales desde la perspectiva de la inferencia de la red y explotando la teoría de la supervivencia. Esta estrategia selecciona los nodos más influyentes tan pronto como se detecta el rumor o cybergossip (con carga negativa o nociva) y lanza una campaña de verdad para crear conciencia contra él, a fin de evitar su impacto e influencia. En consecuencia, se han desarrollado algoritmos basados en el principio de probabilidad, que garantiza una aproximación a los nodos de ciberrumor o cybergossip que predice el 63\% de la solución óptima (Eddine et al., 2020).

Otro elemento a considerar es que la última década ha sido testigo del surgimiento de múltiples plataformas en línea que facilitan a los usuarios obtener ganancias a través de anuncios y venta de productos. Por lo tanto, la búsqueda de nuevas audiencias ha llevado a la creación de títulos de "cebo de clic" para despertar la curiosidad de las personas. Los cyber-rumores y el cybergossip, como declaraciones de información instrumentalmente relevantes, representan la mejor fuente y forma de crear los titulares ideales de "cebo de clic" (Eddine et al., 2020); por ejemplo, titulares de noticias como Cure Cancer Naturally o "Las vacunas 
son peligrosas" se extienden ampliamente en las redes sociales, y varios usuarios creen en estas afirmaciones; al respecto de los rumores en línea sobre las vacunas, estos han reforzado la influencia del movimiento anti-vacunas, convenciendo a más padres sobre el peligro de las mismas.

Otro foco de investigación a destacar en el estudio de la propagación del ciberrumor y el cybergossip en las redes sociales es el problema de maximización de su influencia, pues, al igual que en el gossip, el contenido y la posibilidad de una interpretación nociva incrementa la posibilidad de mayor impacto y alcance en las redes sociales, por lo que el control de rumores se ha convertido en objetivo de varios estudios. Al respecto del control del impacto de estos fenómenos, los esfuerzos se pueden dividir en dos categorías: a) control con estrategias de interrupción de la red y b) estrategias de contrapeso. Las estrategias de interrupción de la red se pueden llevar a cabo eliminando algunos nodos críticos y bordes de la red subyacente que se usan para suprimir la propagación de información errónea. Sin embargo, dicha estrategia no es viable en la práctica ya que interferir con la estructura de la red puede ser imposible para un agente de control y, además, eliminar nodos críticos es una forma de censura que puede violar los estándares éticos, legislativos o reglamentarios en algunos países. Las estrategias de contrapeso, por el contrario, apuntan a reducir la difusión de rumores mediante la difusión de información correcta. En este sentido, investigadores dedicados al estudio del comportamiento on-line en las redes sociales han encontrado en las ciencias sociales computacionales claves relevantes para salvar las limitaciones que los modelos de control de ciber-rumores y cybergossip actualmente tienen; a saber: 1) la poca probabilidad de que un usuario acepte la verdad cuando el ciber-rumor y la verdad lo alcanzan simultáneamente; 2) la probabilidad de que al ser el ciber-rumor y el cybergossip de carga negativa algo más llamativo, una vez que un individuo es activado por este tipo de información, él/ella se mantiene así para siempre y nunca cambia de opinión. Esta última suposición enmarcada en los modelos de difusión competitiva funciona bien para la adopción de productos que se asocia con comportamientos de compra que generalmente no son fácilmente reversibles, pero no para la difusión de información o la formación de opiniones, ya que la actitud de las personas hacia una actividad política o un evento de noticias cambia de acuerdo con la nueva información recopilada de la red (Eddine et al., 2020).

Finalmente, un tercer ámbito que debe incluirse en el estudio del cybergossip es el relacionado con el efecto de proximidad, es decir, el efecto de contraponer la información veraz, "semillas de verdad", en la proximidad de los ciber-rumores o cybergossip (Yang et al., 2020). Esto supone que un individuo que posee óptimas competencias para analizar, filtrar y analizar la información que recibe o encuentra tendrá la tendencia de reconocer y valorar más la veracidad o la verdad de la información y probablemente ignorar el ciberrumor o el cybergossip con carga negativa. Este aspecto conlleva un problema adjunto, el que previa o paralelamente un individuo debe haber aprendido y desarrollado cierto nivel de competencias sociales y emocionales, del pensamiento crítico y además de las competencias digitales (Giardini \& Vilone, 2016; Romera, Cano, García-Fernández, \& Ortega-Ruiz, 2016). 
El panorama es optimista pero, a la vez, retador. En palabras de Meneses (2018), los límites para abordar estos retos complejos se pueden traducir en una colaboración multidisciplinaria, pues requieren una distribución geográfica de su estudio, sustentada en plataformas de colaboración que exigen de la comunidad científica nuevas habilidades de gestión del conocimiento, en lo que Ankey y Leonelli (2016) denominan un nuevo repertorio del cambio científico, que, en síntesis, es una especie de ensamblaje de conceptos, materiales, estrategias, procedimientos y políticas institucionales imprescindibles para la ciencia. Solo desde esta nueva articulación se podrá profundizar en los sentidos del discurso del método digital, que es el resultado de la innegable "datificación" de la sociedad que requiere de novedosas técnicas y métodos para la comprensión de los fenómenos socioculturales que se vienen gestando en las redes sociales $\mathrm{y}$, en general, en internet.

Las reflexiones presentadas en este manuscrito aportan a la comprensión de algunas dinámicas psicológicas en los espacios cibernéticos y sus realidades virtuales, que son un nuevo espacio de transición y extensión del mundo psíquico de persona y grupos, convirtiéndose, por tanto, en el nuevo núcleo de desarrollo de la sociedad, del conocimiento y la información. Así mismo, se ofrece argumentos que pueden beneficiar: a) el desarrollo de programas de formación en competencias digitales, b) la elaboración de proyectos de investigación en ciberconductas, especialmente aquellas que impiden el establecimiento de óptimas relaciones interpersonales, c) el análisis de posibles problemas psicológicos relacionados con el uso de medios digitales, d) la comprensión de las "ciberidentidades", los "sistemas semióticos" y la ciberestética (Colón, 2001); y finalmente, e) el avance general del cuerpo teórico de conocimiento entorno a la ciberpsicología en Colombia.

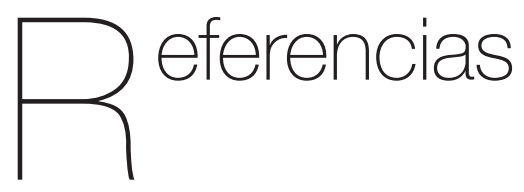

Ankeny, R., \& Leonelli, S. (2016). Repertoires: A post-Kuhnian perspective on scientific change and collaborative research. Studies in History and Philosophy of Science Part A, 60, 18-28. https://doi.org/10.1016/j. shpsa.2016.08.003

Apolloni, B., Malchiodi, D., \& Taylor, J. G. (2013). Learning by gossip: a principled information exchange model in social networks. Cognitive Computation, 5, 327-339. https:// doi.org/10.1007/s12559-013-9211-6

Archer, J. (2004). Sex differences in aggression in real-world settings: ameta-analytic review. Review of General Psychology, 8, 291-322. https://doi.org/10.1037/1089-2680.8.4.291

Archer, J., \& Coyne, S. M. (2005). An integrated review of indirect, relational, and social aggression. Personality and Social Psychology Review, 9, 212-230. doi: 10.1207/s15327957pspr0903_2

Areepattamannil, S., \& Khine, M. S. (2017). Early adolescents' use of information and communication technologies (ICTs) for social communication in 20 countries: Examining the roles of ICT-related behavioral and motivational characteristics. Computers in Human Behavior, 73, 263272. doi: 10.1016/j.chb.2017.03.058 
Baumeister, R. F., Zhang, L., \& Vohs, K. D. (2004). Gossip as cultural learning. Review of General Psychology, 8, 111-121. https:// doi.org/10.1037/1089-2680.8.2.111

Beersma, B., \& Van Kleef, G. A. (2011). How the grapevine keeps you in line: Gossip increases contributions to the group. Social Psychological and Personality Science, 2, 642-649. https://doi. org/10.1177/1948550611405073

Bertolotti, T., \& Magnani, L. (2013). A philosophical and evolutionary approach to cyber-bullying: social networks and the disruption of sub-moralities. Ethics and Information Technology, 15, 285-299. doi: 10.1007/s10676-013-9324-3

Björkqvist, K., Lagerspetz, K. M. J., \& Kaukiainen, A. (1992). Do girls manipulate and boys fight? developmental trends in regard to direct and indirect aggressive behavior. Aggressive Behavior, 18, 117-127. $\quad$ https://doi.org/10.1002/10982337 (1992) $18: 2<117:$ : A I D AB2480180205>3.0.CO;2-3

Brady, D. L., Brown, D. J., \& Liang, L. H. (2017). Moving beyond assumptions of deviance: The reconceptualization and measurement of workplace gossip. Journal of Applied Psychology, 102, 1-25. doi: 10.1037/ apl0000164

Card, N. A., Stucky, B. D., Sawalani, G. M., \& Little, T. D. (2008). Direct and indirect aggression during childhood and adolescence: A meta-analytic review of gender differences, intercorrelations, and relations to maladjustment. Child Development, 79, 1185-1229. http://dx.doi. org/10.1111/j.1467-8624.2008.01184.x
Carrera, A., \& Pelayo, N. (2002). Lenguaje y comunicación: conceptos básicos, aspectos teóricos generales, características, estructura, naturaleza, y funciones del lenguaje y de la comunicación oral y escrita. Venezuela: Los libros de El Nacional.

Chang, L., \& Chen, J. V. (2014). Aligning principal and agent's incentives: A principal agent perspective of social networking sites. Expert Systems with Applications, 41(6), 3091-3104. https://doi.org/10.1016/j. eswa.2013.10.040

Colón, Z. E. (2001). Claves para pensar lo "ciber" en la cibercultura. Designis, 30, 15-22. http://dx.doi.org/10.35659/designis. i30p15-22

Coyne, S.M., Archer, J., \& Eslea, M. (2006). "We're Not Friends Anymore! Unless...": The Frequency and Harmfulness of Indirect, Relational, and Social Aggression. Aggressive Behavior, 32, 294-307. https:// doi.org/10.1002/ab.20126

Cigûenza, N. (2019, Febrero 9). Hasta siete horas puede gastar un colombiano en las redes sociales. La República. Bogotá. Recuperado de https://www.larepublica. co/internet-economy/hasta-siete-horaspuede-gastar-un-colombiano-en-lasredes-sociales-2826041

Davenport, S. W., Bergman, S. M., Bergman, J. Z., \& Fearrington, M. E. (2014). Twitter versus Facebook: Exploring the role of narcissism in the motives and usage of different social media platforms. Computers in Human Behavior, 32, 212-220. doi:10.1016/j. chb.2013.12.011.

Del Rey, R., Casas, J. A., Ortega-Ruiz, R., Schultze-Krumbholz, A., Scheithauer, H., 
Smith, P., ... Plichta, P., (2015). Structural validation and cross-cultural robustness of the European Cyberbullying Intervention Project Questionnaire. Computers in Human Behavior, 50, 141-147. doi: 10.1016/j. chb.2015.03.065

Dunbar, R. I. M. (2004). Gossip in evolutionary perspective. Review of General Psychology, 8, 100-110. https://doi. org/10.1037/1089-2680.8.2.100

Dunbar, R. I. M., Duncan, N. D. C., \& Marriott, A. (1997). Human conversational behaviour. Human Nature, 8, 231-246. https://doi. org/10.1007/BF02912493

Eder, D., \& Enke, J. L. (1991). The Structure of Gossip: Opportunities and Constraints on Collective Expression among Adolescents. American Sociological Review, 56(4), 494508. doi: 10.2307/2096270

Eddine, H. A. I., Li, K., \& Ahmad, S. (2020). Minimizing Rumor Influence in Multiplex Online Social Networks Based on Human Individual and Social Behaviors. Information Sciences, 512, 1458-1480. https://doi. org/10.1016/j.ins.2019.10.063

Ellison, N. B., Vitak, J., Gray, R., \& Lampe, C. (2014). Cultivating Social Resources on Social Network Sites: Facebook Relationship Maintenance Behaviors and Their Role in Social Capital Processes. Journal of Computer-Mediated Communication, 19(4), 855-870, https:// doi.org/10.1111/jcc4.12078

Engelmann, J. M., Herrmann, E., \& Tomasello, M. (2016). Preschoolers affect others' reputations through prosocial gossip. British Journal of Developmental Psychology, 34, 447-460. https://doi.org/10.1111/bjdp.12143
Feinberg, M., Willer, R., \& Schultz, M. (2014). Gossip and ostracism promote cooperation in groups. Psychological Science, 25, 656-664. https://doi. org/10.1177/0956797613510184

Foster, E. K. (2004). Research on Gossip: Taxonomy, Methods, and Future Directions. Review of General Psychology, 8, 78-99. https://doi.org/10.1037/1089-2680.8.2.78

Gabriels, K., \& De Backer, C. J. (2016). Virtual gossip: How gossip regulates moral life in virtual worlds. Computers in Human Behavior, 63, 683-693. https://doi. org/10.1016/j.chb.2016.05.065

Giardini, F. (2012). Deterrence and transmission as mechanisms ensuring reliability of gossip. Cognitive Processing, 13, 465-475. doi: 10.1007/s10339-011-0421-0

Giardini, F., \& Vilone, D. (2016). Evolution of gossip-based indirect reciprocity on a bipartite network. Scientific Reports, 6, 37931. https://doi.org/10.1038/srep37931

Gluckman, M. (1963). Papers in honor of Melville J. Herskovits: Gossip and scandal. Current Anthropology, 4, 307-316. doi: 10.1086/200378

Goodman, M. (2000), Corporate communication: the American picture. Corporate Communications: An International Journal, 5(2), 69-74. https:// doi.org/10.1108/13563280010372496

Grosser, T. J., Lopez-Kidwell, V., \& Labianca, G. (2010). A social network analysis of positive and negative gossip in organizational life. Group \& Organization Management, 35, 177-212. https://doi. org/10.1177/1059601109360391 
Hartung, F. M., \& Renner, B. (2013). Social curiosity and gossip: Related but different drives of social functioning. Plos One, 8, 1-9. doi: 10.1371/journal.pone.0069996

Kapferer, J. N. (1989). Rumores: El medio de difusión más antiguo del mundo. Barcelona: Ed. Plaza y Janés

Kuttler, A. F., Parker, J. G., \& La Greca, A. M. (2002). Developmental and gender differences in preadolescents' judgments of the veracity of gossip. Merrill-Palmer Quarterly, 48, 105-132. https://doi. org/10.1353/mpq.2002.0008

Laghi, F., Schneider, B. H., Vitoroulis, I., Coplan, R. J., Baiocco, R., Amichai-Hamburger, \& Flament, M. (2013). Knowing when not to use the Internet: Shyness and adolescents' on-line and off-line interactions with friends. Computers in Human Behavior, 29, 51-57. http://dx.doi.org/10.1016/j.chb.2012.07.015

Lee, S. L., Kim, J. A., Golden, K. J., Kim, J. H., \& Park, M. S. A. (2016). A Cross-Cultural Examination of SNS Usage Intensity and Managing Interpersonal Relationships Online: The Role of Culture and the Autonomous-Related Self-Construal. Frontiers in Psychology, 7, 1-12. https://doi. org/10.3389/fpsyg.2016.00376

Lila, M., Musitu, G., \& Buelga, S. (2000). Adolescentes colombianos y españoles: diferencias, similitudes y relaciones entre la socialización familiar, la autoestima y los valores. Revista Latinoamericana de Psicología, 32, 301-319. Recuperado de https://www.redalyc.org/ pdf/805/80532203.pdf

Litman, J. A., \& Pezzo, M. V. (2005). Individual differences in attitudes towards gossip. Personality and Individual Differences,
38, 963-980. http://dx.doi.org/10.1016/j. paid.2004.09.003

Livingstone, S., Haddon, L., Vincent, J., Mascheroni, G., \& Ólafsson, K. (2014). Net Children Go Mobile: The UK Report. London: London School of Economics and Political Science.

López-Pradas, I. C., Romera, E. M., Casas, J. A., \& Ortega-Ruiz, R. (2017). Cybergossip and cyberbullying during primary school years. Psicología Educativa, 23(2), 73-80. http://dx.doi.org/10.1016/j.pse.2017.05.007

Mason, K. L. (2008). Cyberbullying: A preliminary assessment for school personnel. Psychology in the Schools, 45, 323-348. http://dx.doi.org/10.1002/ pits.20301.

Mascheroni, G., \& Cuman, A. (2014). Net Children Go Mobile: Final report (Deliverables D6.4 \& D5.2). Milano: Educatt.

Martinescu, E., Janssen, O., \& Nijstad, B. A. (2014). Tell me the gossip: The selfevaluative function of receiving gossip about others. Personality and Social Psychology Bulletin, 40, 1668-1680. https:// doi.org/10.1177/0146167214554916

Massar, K., Buunk, A. P., \& Rempt, S. (2012). Age differences in women's tendency to gossip are mediated by their mate value. Personality and Individual Differences, 52, 106-109. https://doi.org/10.1016/j. paid.2011.09.013

McAndrew, F. T. (2014). The "sword of a woman": Gossip and female aggression. Aggression and Violent Behavior, 19, 196199. doi: 10.1016/j.avb.2014.04.006 
McDonald, K. L., Putallaz, M., Grimes, C. L., Kupersmidt, J. B., \& Coie, J. D. (2007). Girl talk: Gossip, friendship, and sociometric status. Merrill-Palmer Quarterly, 53, 381-411. https://doi.org/10.1353/ mpq.2007.0017

Meneses, M. H. (2018). Grandes datos, grandes desafíos para las ciencias sociales. Revista Mexicana de Sociología, 80(2), 415-444. http://dx.doi.org/10.22201/ iis.01882503p.2018.2.57723

Merten, D. E. (1997). The meaning of meanness: Popularity, competition, and conflict among junior high school girls. Sociology of Education, 70, 175-191. doi:10.2307/2673207

Nevo, O., Nevo, B., \& Derech-Zehavi (1993). The development of the Tendency to Gossip Questionnaire: Construct and concurrent validation for a sample of Israeli college students. Educational and Psychological Measurement, 53, 973-981. doi: 10.1177/0013164493053004010

Noon, M., \& Delbridge, R. (1993). News from behind my hand: Gossip in organizations. Organization Studies, 14, 23-36. https:// doi.org/10.1177/017084069301400103

Oh, H. J., Ozkaya, E., \& LaRose, R. (2014). How does online social networking enhance life satisfaction? The relationships among online supportive interaction, affect, perceived social support, sense of community, and life satisfaction. Computers in Human Behaviors, 30, 69-78. https://doi. org/10.1016/j.chb.2013.07.053

Oluwole, D. A. (2009). Spirituality, gender and age factors in cybergossip among Nigerian adolescents. Cyberpsychology \& Behavior,
12(3), 323-326. http://dx.doi.org/10.1089/ cpb.2008.0285

Perry, D. G., \& Pauletti, R. E. (2011). Gender and adolescent development. Journal of Research on Adolescence, 21, 61-74. http://dx.doi. org/10.1111/j.1532-7795.2010.00715.x

Peter, F. (2013, Abril 23). 'Bogus' AP tweet about explosion at the white house wipes billions off US markets, The Telegraph, Finance/Market, Washington. Retrieved from https://www.telegraph.co.uk/finance/ markets/10013768/Bogus-AP-tweetabout-explosion-at-the-White-Housewipes-billions-off-US-markets.html

Peters, K., \& Kashima, Y. (2015). Bad habit or social good? How perceptions of gossiper morality are related to gossip content. European Journal of Social Psychology, 45, 784-798. https://doi.org/10.1002/ejsp.2123

Pietrosemoli, L. (2009). El chisme y su función en la conversación. Lengua y Habla, 13, 5567. Recuperado de https://www.redalyc. org/pdf/5119/511951369005.pdf

Reinecke, L., \& Trepte, S. (2014). Authenticity and well-being on social network sites: A two-wave longitudinal study on the effects of online authenticity and the positivity bias in SNS communication. Computers in Human Behavior, 30, 95-102. https://doi. org/10.1016/j.chb.2013.07.030

Romera, E. M., Cano, J. J., García-Fernandez, C. M., \& Ortega-Ruiz, R. (2016). Cyberbullying: social competence, motivation and peer relationships. Comunicar, 24, 71-79. https:// doi.org/10.3916/C48-2016-07

Romera, E. M., Herrera-López, M., Casas, J. A., Ortega, R. \& Del Rey, R. (2018). How 
Much Do Adolescents Cybergossip? Scale Development and Validation in Spain and Colombia. Frontiers in Psychology, 9, 126. doi: 10.3389/fpsyg.2018.00126

Said-Hung, E. (2014). Jóvenes vulnerables y participación móvil en Colombia: estudio sobre el grado de participación y apropiación ciudadana entre beneficiarios de programas sociales. Innovar, 24, 31$44 . \quad$ https://doi.org/10.15446/innovar. v24n52.42504

Schindler, M. P. (2003). Rumors in Financial Markets: Survey on how they evolve, spread and are traded on. Institute for Empirical Research in Economics, Universidad de Zurich. Retrieved from http://pascalfroissart. online.fr/3-cache/2003-schindler.pdf

Smith P. K. (2006). Ciberacoso: naturaleza y extensión de un nuevo tipo de acoso dentro y fuera de la escuela. Trabajo presentado al Congreso Educación de Palma de Mallorca. Recuperado de https://studylib. es/doc/4921324/ciberacoso1--naturalezay-extensi\%C3\%B3n-de-un-nuevo-tipode-a...

Stirling, R. B. (1956). Some psychological mechanisms operative in gossip. Social Forces, 34, 262-267. https://doi. org/10.15446/innovar.v24n52.42504

Subramanian, M. (2013). Gossip, drama, and technology: How South Asian American young women negotiate gender on and offline. Gender and Education, 25, 311324. http://dx.doi.org/10.1080/09540253.2 012.746647

Tafarodi, R. W., \& Swann, W. B. (1996). Individualism-collectivism and global self-esteem. Journal of Cross-Cultural
Psychology, 27, 651-672. https://doi. org/10.1177/0022022196276001

Tinoco, C. E. (2004). Dinámica del rumor y operaciones psicológicas de daño reputacional. Anales de la Universidad Metropolitana, 4(2), 155-169.

Tokunaga, R. S. (2010). Following you home from school: A critical review and synthesis of research on cyberbullying victimization. Computers in Human Behavior, 26, 277-287. http://dx.doi.org/10.1016/j. chb.2009.11.014.

Watson, D. C. (2012). Gender differences in gossip and friendship. Sex Roles, 67, 494-502. https://doi.org/10.1007/ s11199-012-0160-4

Wert, S. R., \& Salovey, P. (2004). A social Comparison Account of Gossip. Review of General Psychology, 8, 122-137. https://doi. org/10.1037/1089-2680.8.2.122

Wu, J., Balliet, D., \& Van Lange, P. A. (2016). Reputation management: Why and how gossip enhances generosity. Evolution and Human Behavior, 37(3), 193-201. https://doi. org/10.1016/j.evolhumbehav.2015.11.001

Xie, H., Cairns, B. D., \& Cairns, R. B. (2005). The development of aggressive behaviors among girls: Measurement issues, social functions, and differential trajectories. In D. J. Pepler, K. C. Madsen, C. Webster, \& K. S. Levene (Eds.), The development and treatment of girlhood aggression (pp. 105-136). Mahwah, NJ: Lawrence Erlbaum Associates.

Yang, L., Li, Z., \& Giua, A. (2020). Containment of rumor spread in complex social networks. Information Sciences, 506, 113-130. https:// doi.org/10.1016/j.ins.2019.07.055 